\title{
SUR L'UNIVERS FANTASTIQUE \\ DE JULIO CORTAZAR: \\ ANALYSE SEMANTIQUE DE LA \\ REFERENCIATION DANS PASAJES ${ }^{1}$
}

Alma Rosa Aguilar Gutiérrez
Universidad Nacional, Costa Rica

\section{Introduction}

L'objet du présent travail est d'étudier la problématique interprétative posée par un ensemble de récits fantastiques: quatre nouvelles rassemblées dans le volume Pasajes de l'écrivain argentin Julio Cortázar. En 1976, il a réorganisé en quatre volumes thématiques ses nouvelles publiées auparavant. Nous analysons les parcours interprétatifs susceptibles d'être suivis par le lecteur d'après la programmation suggérée par ces textes ainsi quel'activité conceptuelle et langagière de production et de compréhension du sens à laquelle participent scripteur et lecteur. Du point de vue de la conceptualisation et de la construction de la référence au temps, à l'espace et à la personne, nous caractérisons les marques linguistiques, les indices d'information extralinguistique et les organisations discursives mises en oeuvre par le scripteur pour faire de ces domaines référentiels, des

1. Síntesis de lo expuesto en la tesis La Référenciation dans la construction d'un récit fantastique, presentada en la Universidad de Paris X, Nanterre, para optar al grado de Doctorado en Letras, 1998. 
matériaux du fantastique. Du point de vue du lecteur, nous examinons l'activité de construction de la signification; il s'agit d'identifier les mécanismes sollicités pour cette activité.

\section{Cadre théorique}

Le cadre théorique adopté tient compte de la complexité de l'interaction scripteur - texte- lecteur et de la dimension cognitive dans l'activité de compréhension. Unesémantique textuelle à dimension interprétative nous a semblé appropriée pour aborder la référenciation dans les trois domaines conceptuels qui organisent ces récits: temps, espace, personne. Nous avons rassemblé différentes recherches sur l'approche sémantique des textes effectuées dans le domaine de la linguistique et de la psychologie cognitive. Afin de construire un corps conceptuel cohérent et un parcours méthodologique raisonné, nous avons explicité, dans le cadre des théories d'où ils sont issus, certains concepts opératoires qui nous semblaient essentiels, pertinents et compatibles du point de vue de leurs perspectives d'investigation et de leur mise en oeuvre sur des textes.

\section{Corpus}

Les opérations de référenciation au temps comme matériau du fantastique sont examinées dans «El otro cielo». La référence à la personne estétudiée à partir de «Lejana». L'importance prépondérante de la spatialité dans la construction de la figure du passage, a motivé un examen de quatre nouvelles: «Ahí pero dónde», cómo?», «La isla al mediodía», «Lejana» et «El otro cielo»².

2. Toutes les citations de Cortázar renvoeint à l'édition Los relatos 3. Pasajes (Madrid: Alianza, 1985). 


\section{Démarche d'analyse}

La démarche d'analyse est fondée sur une sémantique textuelle ct interprétative qui vise à considérer le cognitif et le linguistique et comprend deux niveaux d'organisation sémantique du texte. Ainsi, la construction d'une signification locale (microstructre) et globale (macrostructure), sont analysées en détail.

Le niveau microstructurel: la séquence narrative constituel'unité d'analyse, comme niveau intermédiaire à l'intérieur de laquelle nous faisons une microanalyse de sémantique des prédications et des procès, les chaînes anaphoriques, la deixis pour la référence à la personne et ainsi de suite pour toutes les opérations de référenciation au temps, à l'espace et à la personne.

Le niveau macro-structurel comprend une présentation de la nouvelle, de la macro-structure sémantique du texte, de la structure discursive et de la perspective narrative.

\section{Le temps materiel du fantastique: «El otro cielo»}

Porque hubo una época en que las cosas me ocurrían cuando menos pensaba en ellas, empujando apenas cualquier rincón de aire, El otro cielo, Julio Cortázar.

Les travaux en sémantique textuelle de Colette Noyau sur le temps dans la nouvelle latinoaméricaine inspirent notre démarche. L'étude de Jacques François sur l'articulation chronologique du récit influence aussi les analyses ${ }^{3}$. Nous visons à élucider deux aspects: la construction de la temporalité par le scripteur et l'activité du lecteur

3. Voir particulièrement de Colette Noyau, «Le temps retourné: Viaje a la semilla, de Alejo Carpentier», in Gilles Luquet (éd.)Actes du Quatrième Colloque de Linguistique Hispanique (Limoges: Presses Universitaires de Limoges, 1992)et de Jacques François: «La compréhension de l'articulation chronologique des séquences narratives: un exercice de linguistique cognitive», Verbum 4 (1993) 49-70. 
pour parvenir à l'interprétation du sens. Notre analyse a montré: les moyens linguistiques mis en oeuvre pour produire l'imbrication des éléments doubles, au niveau de l'histoire, du temps et de l'espace; la nature du caractère fantastique du récit; le parcours de lecture proposé par le texte ainsi que les contraintes auxquelles le lecteur doit faire face dans son parcours interprétatif.

$\mathrm{Au}$ niveau micro-sémantique nous avons étudié l'ancrage temporel: les opérations linguistiques de référenciation temporelle, et la représentation des procès. Les procès caractéristiques d'un traitement particulier sont analysés à partir de leurs traits sémantiques. Enfin, nous recapitulons l'ensemble des moyens linguistiques temporels intervenant dans les jeux avec le temps qui se trouvent dans le texte.

«El otro cielo» («L'autre ciel») présente un personnage qui raconte deux époques de sa vie, des expériences d'adolescence et sa vie actuelle, et la rencontre avec une prostituée; situées à Buenos Aires et à Paris respectivement. Les événements sont disposés dans une suite logique normale, et l'évocation de l'histoire de Josiane, la prostituée, occupe presque la totalité du récit, constitue une sorte de premier plan.

Cette nouvelle construit deux mondes et deux histoires indépendantes qui s'entrecroisent à l'intérieur de la trame narrative. De nombreux éléments opposent ces deux mondes dont le rapprochement se fait par le biais du passage. Le passage constitue une irruption du fantastique dans le quotidien, une altération momentanée, ponctuelle, de la regularité du quotidien.

\section{L'information temporelle: le temps des histoires}

Les problèmes de temporalité sont particulièrement complexes dans l'univers fantastique Cortazarien. La nouvelle a une structure temporelle dont le cadre initial est donnée par l'emploi des formes verbales au présent dans la séquence d'ouverture, situant le moment de la parole au même intervalle que certains événements racontés dans l'histoire qui se déroule à Buenos Aires (HBA). Le site temporel du 
présent est ici défini par rapport à un repère absolu, une date du calendrier: Hacia el año veintiocho fixe le temps de référence initial (TR1) de l'univers fictif de HBA. L'absence de marqueurs chronologiques explicites dans les épisodes suivants créent le flou et l'ambiguité en ce qui concerne l'intervalle où se déroule HBA.

L'information temporelle qui marque l'intervalle occupé par chaque histoire dans l'axe temporel est donnée par des références historiques. On peut reconstruire un axe chronologique pour HBA à partir de trois moments: le dénouement de la guerre mondiale, l'avancement des troupes alliées, la libération des capitales cruropéennes et la bombe sur Hiroshima. Ces informations ne sont fournies qu'au fil du quatrième épisode, ce qui laisse supposer qu'une bonne partie du parcours de la lecture se fait sur la base de l'hypothèse temporelle initiale (autour de 1928). La localisation temporelle de l'histoire qui se déroule à Paris (HP) est indirecte et relève de l'ordre de l'inférence, elle peut être reconstruite à partir d'indices lexicaux: «vela», «cochero», «sombrero de copa», «fiacre», «prusianos», et «yesquero».

\section{Les moyens linguistiques}

L'analyse des valeurs des temps verbaux révèle une complexité particulière dans leur emploi, le présent de l'énonciation est souvent suivi d'un présent de narration et du discours direct au présent. L'exploitation des différentes valeurs du présent ainsi que le choix systématique de l'infinitif et du gérondif, constitue un mécanisme pour effacer le barrières temporelles et créer une impression de simultanéité et continuité entre les événements et les situations, bref une façon de programmer une lecture linéaire.

Les circonstants temporels fonctionnent comme marqueurs d'ordre chronologique et situent les événements dans un laps de temps qui définit la durée de la période, mais sans en fixer le site temporel exact: en esos días, en esa época. Era la época, a principios de un invierno, Hasta que otra vez. Ces marqueurs indiquent vaguement le 
temps des événements, et permettent de jouer avec des époques très éloignées dans le temps, et tant qu'elles ne sont pas explicitées et reconnues pour le lecteur, le récit paraît cohérent. Par ailleurs, ces indices marquent une progression dans l'histoire racontée: Más tarde hubo otras cosas et la coexistence des histoires à une même époque en esa época mi madre...

La durée des macro-situations de l'histoire qui se déroule à Paris est déterminée de façon globale et imprécise, par «quelques semaines,»Hubo algunas semanas».

\section{L'imbrication de deux histoires}

Un procédé d'imbrication au niveau des séquences narratives impliquant les histoires, le temps et l'espace est à la base du fantastique dans cette nouvelle. L'articulation chronologique y joue un rôle central dans la construction d'une représentation globale de la temporalité et de la siginification en général.

En absence d'un marquage chronologique explicite, d'autres éléments linguistiques jouent le rôle de marqueurs. Les temps verbaux établissent les relations temporelles entre situations et événements. L'ordre de succession des prédications temporalisées dans une séquence correspond à l'ordre d'occurrence des procès relatés. Un premier exemple du procédé d'ímbrication des deux histoires: «Irma es la más buena y generosa de las mujeres [...] Supongo que por cosas así acabé conociendo a Josiane (p. 15).

La local isation temporelle est donnée par l' information contenue dans les prédications juxtaposées qui s'enchaînent logiquement sur un axe temporel défini. Les déterminations anaphoriques marquent aussi le rapportexistant entre les événements ainsi que la valeur de causativité de marqueurs directs de postérité par «por». Le lecteur peut construire des hypothèses sur les relations de causalité et d'antériorité-postériorité entre les états, les événements et de continuité entre les séquences. Notons que dans cette séquence il y a une articulation au niveau 
discursif des valeurs de premier et d'arrière plan qui donne l'impression d'un récit parfaitement cohérent.

Les opérations de référenciation temporelle qui organisent le récit et autorisent le lecteur à interpréter l'ordre des événements sont stables pendant un tiers de la nouvelle. Cependant, elles vont entrer en contradiction avec les nouvelles références temporelles qui délimitent l'intervalle temporel de l'histoire, en fixant ainsi le cadre temporel global et en établissant l'intervalle qui correspond aux événements de chaque univers. Dans un premier temps, l'organisation de l'information autorise à localiser les états et les événements dans des intervalles d'un axe temporel, sans préciser le temps chronologique qui les sépare. Le lecteur, avec son expérience du cours des choses, reconstruit ce qui est dit dans le texte et formule des hypothèses pour relier (par une relation de simultanéité ou de succession) les situations avec les événements et les situer à une même époque. L'hypothèse construite à partir de la temporalité grammaticale et les marqueurs indiqués sera valable jusqu'à ce que d'autres signes linguistiques viennent la rendre caduque. L'ordre chronologique d'ocurrence des événements auquel le lecteur est habitué est bousculé par des informations temporelles fournies ultérieurement. Le lecteur doit entreprendre une nouvelle tâche, réinterpréter le sens du texte en tenant compte d'un réseau complexe d'informations temporelles qui concourent directement ou indirectement à l'articulation chronologique du récit.

\section{Les jeux avec le temps}

Le jeu au niveau conceptuel avec le temps consiste à établir des relations d'antériorité, de postériorité, de simultanéité et de causalité contradictoires. On construit une chaîne consécutive des événements situés à des intervalles très éloignés sur un axe temporel ordinaire. Dans un premier temps, le lecteur fait un repérage linguistique qui l'autorise à se construire une représentation temporelle de l'ordre d'occurrence des événements qui sera bousculée par les nouveaux 
indices dont la valeur en tant que marqueurs chronologiques fixe d'autres repères temporels. D'après l'expérience du lecteur, la reconstruction d'une succession temporelle chronologique ordinaire tenant compte des repères contradictoires lui est impossible. Il est face à deux interprétations chronologiques en concurrence. Le jeu consiste à faire intervenir dans le texte deux procédés différents de construction de la temporalité. L'un répond à un système de repères linguistiques qui autorise une construction du cheminement temporel et l'autre renvoie à la valeur chronologique absolue sur une échelle de temps socialisé qui fixe de façon incontestable le temps de référence.

Les opérations de marquage des relations temporelles et de causalité caractérisent le procédé d'imbrication des événements à l'intériéur des séquences narratives. C'est au moyen d'une analyse sémantique détaillée qu'on peut interpréter ces phénomènes de rétrocession dans le temps et de cohérence apparente qui font de ce domaine d'expérience un matériau du fantastique.

Voyons maintenant une séquence où les marques de temps grammaticaux fonctionnent comme marqueurs d'articulation chronologique. Al'intérieur de cette séquence, on trouve deux situations introduites par les énoncés que nous distinguons par A et B:

(A) Sé muy bien que jamás se le ocurriría contárselo a Irma, pero lo mismo me fastidia la persistencia de un derecho materno que ya nada justifica, y sobre todo que sea yo el que al final se aparezca con una caja de bombones o una planta para el patio, y que el regalo represente de una manera muy precisa sobreentendida la terminación de la ofensa, el retorno a la vida corriente del hijo que vive todavía en casa de su madre.

(B) Desde luego Josiane era feliz cuando le contaba esa clase de episodios, que una vez en el barrio de las galerías pasaban a formar parte de nuestro mundo con la misma llaneza que su protagonista (p. 16). 
Dans ce paragraphe, certaines prédications vont retenir notre attention: Je sais... introduit une prédication d'état psychologique qui inclut toute la durée de l'histoire et qui marque un temps de référence par rapport au moment de l'énonciation. Le statut du présent dans cette séquence pourrait être perçu comme ambigu. D'une part, on pourrait penser qu'il s'agit d'un présent de narration et que le contenu des énoncés est seulement valable pour le moment de la situation. Mais d'autre part, étant donné que le sémantisme des verbes relève de l'ordre de la connaissance, c'est-à-dire du faire cognitif: «je sais, je déteste, rien ne justifie», rien n'indique que ces informations ne soient pas encore valables au moment de l'énonciation. Par conséquent, l'exploitation de différentes valeurs des temps grammaticaux ainsi que le sémantisme des lexèmes contribuent à forger l'incertitude: le présent ne renvoie pas toujours, dans cette nouvelle, au même temps de référence.

Dans cet exemple, la marque de temps grammatical des forme: era, contaba et pasaban fonctionne comme marqueur d'articulation chronologique appartenant à la catégorie du Temps grammatical (imparfait) et exerçant une fonction à la fois temporelle et aspectuelle. En outre, le sens du verbe pasaban et son environnement actantiel renforcent l'articulation chronologique. Le rôle des indices est tout à fait clair à cet égard: esa clase de episodios que una vez en el barrio de las galerías pasaban a formar parte de nuestro mundo. Cette prédication de changement locatif a pour fonction de fusionner les deux mondes en les articulant aux niveaux discursif et sémantique.

La même fonction d'enchaînement événementiel est accomplie par le déterminant esa clase. Suivant Weinrich, dans le cadre d'une sémantique textuelle la valeur du signal «esa» est importante pour la détermination de la place de l'information ${ }^{4}$. Esa clase de episodios réfère à un épisode situé comme antérieur à l'assertion par son mode

4. Harald Weinrich, Le temps (Paris: Seuil, 1973). 
de détermination. Ce déterminant marque donc une relation de succession entre un épisode et une situation localisée dans un intervalle contigu sur l'axe avant-après.

De cette façon, on a vu un exemple où le narrateur ramène des faits qui se passent dans le monde réel au monde évoqué, ce qui vient perturber l'ordre chronologique d'ocurrence des événements auquel le lecteur est habitué. Les relations interévénementielles reliant les deux espaces proposés par le récit contredisent cet ordre chronologique. Le narrateur joue avec le temps en se servant aussi des temps verbaux, et ce n'est qu'au moyen d'une analyse ponctuelle qu'on peut interpréter ces phénomènes de rétrocession et glissement dans le temps. En procédant à cette reconstruction de ce qui est dit par le texte, on parvient à rendre compte d'une figure narrative cruciale: le jeu avec le temps, l'inversion temporelle en l'occurrence.

En ce qui concerne les phrases que nous avons commentées (A et $\mathrm{B}$ dans l'exemple ci-dessus), elles sont disposées à l'intérieur d'un ensemble de propositions narratives qui sont censées appartenir à une même séquence. D'autre part, (B) possède un connecteur chronologique: cuando. Cet articulateur fixe le moment de déclenchement du procès introduit antérieurement. Le repérage de ces éléments permet d'effectuer une inférence temporelle, et donc de situer les deux procès relatés ainsi en juxtaposition dans des intervalles consécutifs sur un même axe temporel. «B» est alors interprété comme postérieur à «A» mais proche dans une relation de continuité. Ces moyens lexicaux encouragent les inférences référentielles qui autorisent le lecteur à établir des rapports entre les énoncés, et assurent la cohérence logico-sémantique du texte du point de vue de la successsion des événements. Du point de vue linguistique, le texte produit un effet d'articulation temporelle au moyen de marqueurs indirects fixant une relation chronologique en termes de logique des relations entre les événements. Pour ce qui est de l'histoire racontée, le lecteur commence à construire une hypothèse sur la simultanéité de l'existence des acteurs (la mère, Irma, Josiane) à une même époque. 
Cette analyse a permis aussi d'observer le mode de coopération entre deux stratégies d'ordre linguistique et conceptuel nécessaire pour la compréhension du cheminement temporel dans «El otro cielo».

\section{La référénce a la personne: «Lejana»}

«Pero qué tc pasa?» Le pasaba a aquella, a mí tan lejos», «Lejana», Julio Cortázar.

Dans «Lejana» la référence à la personne constitue le mécanisme essentiel de construction du fantastique. Cette nouvelle est structurée à partir de la problématique de l'altérité, figure dominante du recueil Pasajes. La nouvelle a la forme d'un journal intime. Il s'agit du parcours d'une femme à travers ses pensées; elle vit des expériences extraordinaires et progressivement prend conscience de l'existence de son double, d'une autre partie d'elle-même, quelque part dans le monde, très loin de chez elle. A la fin, elle décide de partir à la recherche d'elle-même.

«Lejana» est une des nouvelles les plus réussies sur le thème du double par la nature du parcours de fusion des personnalités. Ce parcours implique la sélection minutieuse des éléments susceptibles de rendre compte, de façon précise, d'une activité mentale et sensorielle intense.

Les parcours sémantiques programmés par le texte visent à faire construire une réprésentation de cette entité binaire, composée de deux êtres qui vivent simultanément deux histoires différentes localisées dans deux espaces très éloignés l'un de l'autre: Buenos Aires et Budapest. Les jeux au niveau du conceptuel et du langagier caractérisent le processus de constitution de cette entité; évidemment, cette nouvelle conception de la nature humaine a des implications lors de la mise en discours et oblige par conséquent à un traitement du langage qui viole les lois ordinaires d'agencement des éléments linguistiques. L'analyse sćmantique des opérations de référenciation à la personne a permis de décrire le procédé de création du fantastique qui fonctionne dans cet univers clos et singulier créé par ce texte. 
Le sens de l'énoncé, est étudié par rapport aux relations qu'il entretient avec d'autres éléments linguistiques et conceptuels au niveau de la séquence ainsi que les implications au niveau de la construction de la macrostructure sémantique.

\section{Deixis, anaphores et chaînes anaphoriques dans la construction de l'identité}

Les opérations de référenciation à la personne suivent d'autres contraintes que celles qui régissent une narration de type factuel réel. Un système référentiel particulier est mis en place pour introduire un deuxième personnage,(le double). Cette deuxième entité est introduite par des procédés transgressant les contraintes qui régissent la référenciation à la personne dans un texte narratif, et en particulier une nouvelle entité. La création d'un texte cohérent impliquerait un mouvement référentiel régi par des contraintes spécifiques qui obligent la sélection de marques linguistiques déterminées. L'introduction des informations avec des déterminants fonctionne avec une logique interne particulière. On les présente comme si c'était de l'information connue:

Horrible porque abre camino a esta que no es reina y que otra vez odio de noche. A esa que es Alina Reyes pero no la reina del anagrama. Que será cualquier cosa (p. 91).

De manière générale (sauf dans les incipits «in medias res» produisant un effet spécial) dans les narrations, l'emploi de la détermination suppose qu'on fait réference à un élément déjà connu dont l'antécédent se trouve en amont et cela fait supposer qu'on est face à de l'information déjà donnée dans le texte. Or ce n'est pas le cas dans la séquence ci-dessus. Esta «celle» ou ésa «celle-ci» introduisent un nouveau personnage. L'emploi de ces mécanismes atteste d'une volonté, de la part du scripteur, de créer une ambiguïté, et ne paraît pas 
refléter la mise en discours de représentations mentales précises en ce qui concerne les entités.

Le texte propose, en principe, la construction de deux représentations mentales parallèles d'entités, à partir des parcours des marques linguistiques de flexions verbales et pronominales qui renvoient respectivement à la première et à la troisième personne du singulier. C'est à partir de ces indices initiaux, que sont classées les références ultérieures.

L'information autorisant la construction de la deuxième entité avait été introduite à la fin du premier épisode par une série d'énoncés offrant des caractéristiques qui la mettent dans une situation défavorisée, fragile, indeterminée et d'éloignémant. Le point de vue adopté est celui du protagoniste qui, dès le départ, se situe au-dessus de l'autre en marquant une distinction par rapport à la lointaine. Les énoncés qui suivent, bien qu'ils suggèrent l'identification de celle-ci à Alina Reyes, font supposer que le sujet de l'énoncé et le sujet de l'énonciation sont distincts. En principe, c'est vrai, sauf que celle qui énonce (celle qui écrit) s'appelle Alina Reyes, et dans son énonciation identifie une autre Alina Reyes. Ce jeu n'est pas répérable à une première lecture: A esta + no reina + cualquier cosa + mendiga + pupila de mala casa + sirvienta, A esa + que es Alina Reyes pero + no la reina del anagrama + lejos.

L'emploi de ces démonstratifs référant à une nouvelle entité peut s'expliquer par ce que Kleiber appelle la «deixis textuelle» ${ }^{5}$. Les démonstratifs peuvent être intégrés à la classe des déictiques (pour marquer leur identité sémantico-référentielle) si on ajoute au cadre déictique formé par le moment, l'endroit et les participants les objets résidant dans la situation. "Ésta» et «ésa», sont des expressions déictiques qui renvoient à des entités dont le dénominateur commun est qu'ils sont localisés dans la situation d'énonciation. Dans ce cas

5 Georges Kleiber, «Déictiques, «token-reflexives», symboles indexicaux, etc.: comment les définir», Cahier de linguistique française $(14,1986) 104$. 
précis «ésta»: celle-ci et «ésa» celle-là ne peuvent pas être interprétés comme des expressions anaphoriques puisqu'elles ne renvoient pas à un segment linguistique plus ou moins proche, ce qui constitue le caractère spécifique de l'anaphore. L'environnement contextuel (la situation initiale du récit) fait penser plutôt à un emploi déictique en ostention sur la personne (imaginaire dans ce cas). Cependant, on peut parler de «déictique opaque» puisque la situation initiale telle qu'elle est présentée ne dissipe pas l'opacité référentielle dans un premier temps. Or, une relecture permet d'analyser le rôle des déictiques textuels. En réalité, il s'agit d'un effet spécial qui favorise la construction d'une représentation mentale de la situation intégrant une autre entité (réelle ou imaginaire) sur la scène; cela permet de donner un caractère d'étrangeté à cette entité qui vient s'installer dans l'espace quotidien de la protagoniste par des moyens extraordinaires. Evidemment une première lecture ne permet pas de saisir des indices aussi subtils mais introduit déjà le lecteur dans un territoire confus, incertain.

La référenciation à la personne est très complexe. Le rapport entre les deux entités telles qu'on les présente linguistiquement n'est pas clair. Au fur et à mesure que l'histoire progresse, l'imbrication des entités se fait plus évidente, même au niveau de l'énoncé. L'ambiguité référentielle se manifeste dans une fusion des entités par une opération de tressage au niveau de la signification et de la définition du protagoniste:

Puedo solamente odiarla tanto, aborrecer las manos que la tiran al suelo y también a ella, a ella todavía más porque le pegan, porque soy yo y le pegan... Que sufra; que se hiele. Yo aguanto desde aquí y creo que entonces la ayudo un poco (p. 91).

Les deux entités représentées par les expressions référentielles «Je» et «elle» accomplissent ou subissent l'action, leur référent est le même: la personne d'Alina Reyes introduite par deux pronoms, en principe contradictoires pour référer à la même entité. Cette 
impossibilité grammaticale d'identification du sujet sur le plan réel nous lance dans l'univers textuel fictionnel où l'altérité serait un phénomène sinon ordinaire du moins possible.

L'appuisurl'information contextuelle est absolument nécessaire pour l'interprétation de certains énoncés, qui jouent un rôle crucial dans l'évolution de l'histoire et marquent les moments de fusion des deux entités. Dans ce cas, la valeur de la conjonction «et», qui en principe lie deux énoncés de même nature vient, de manière subversive, ćtablir un rapport d'équivalence conceptuelle entre «elle et moi». La fusion des entités se fait par l'emploi d'opérations de référenciation subversive. Le sens est construit au moyen d'énoncés juxtaposés entre lesquels il faut postuler des relations sémantiques, de causeconséquence et de continuité.

\section{Les rôles actanciels}

L'identification des rôles actanciels accordés à chacun des personnages est déterminante pour le travail de coopération interprétative dans cette nouvelle. Tout au long du texte, on assigne différents rôles à chaque personnage, deux rôles à la protagoniste et un seul à la lointaine. Une analyse des prédications détermine le statut des différents personnages. Dans les prédications attribuant une action, un état ou un processus à Alina, elle assume souvent un rôle d'expérienceur. Le sujet grammatical renvoyant à la protagoniste, d'après le constituant sémantique participatif des prédications, joue un rôle actanciel caractérisé par des traits: agent, expérienceur, contrôleur. Elle contrôle les actions concernant la prise de décisions, consciente de sa dualité, elle domine l'histoire. L'autre partie d'Alina exécute des actions sans transcendance.

L'interprétation du dénouement de l'histoire dans «Lejana» exige la mise en oeuvre d'une stratégie linguistique pour distinguer les deux chaînes anaphoriques renvoyant aux différentes parties mais complémentaires de la protagoniste. Il est important de: 
- suivre la piste des rôles de contrôleur et d'expérienceur du personnage pour inférer que le personnage qui reste sur le pont est celui qui a maintenu ce rôle d'expérienceur dans les séquences de prise de contact avec la lointaine.

- Saisir un réseau sémantique des rapports entre les rôles assumés par le protagoniste tout au long du texte et culmine dans la séquence finale.

- La fin de l'histoire reste focalisée sur le sujet expérientiel; la partie dominante du personnage Alina qui perçoit son double, c'est la même qui continue à jouer le rôle d'expérienceur sur le pont. L'analyse des prédications dénote qu'elle s'approprie totalement le corps et la souffrance de la mendiante.

\section{L'espace}

La spatialité est étudiée dans quatre nouvelles «L'autre ciel», «La lointaine», «L'île à midi», «Là mais où et comment».

\section{Démarche d'analyse}

Une fois déterminées les particularités qui caractérisent le traitement langagier opéré dans le texte pour construire l'espace où s'insère l'histoire, ainsi que les relations qui unissent les configurations spatiales entre elles et aux autres composants du récit: temps, personnages et actions, nous avons étudié la signification et la valeur symbolique de l'exploitation combinée de l'ici et de l'ailleurs, du clos et du fermé, du réel et de l'iréel. Notre but a été de répondre à des questions: par quels moyens le scripteur construit-il le monde? Comment parvient-il à une conceptualisation particulière des espaces fantastiques? Comment le lecteur peut-il capter les indices dans le texte pour saisir la représentation de l'espace conçue par le scripteur?

Pour y répondre, nous avons fait une étude du dispositif de la spatialisation du récit à partir d'une analyse du travail linguistique et 
(onceptuel mis en oeuvre dans la création d'un univers, qui dépasse le niveau phrastique. Cette étude a pour but de découvrir l'ensemble de parcours sémantiques à travers lesquels l'espace est constitué comme un matériau du fantastique. Ensuite nous avons analysé les rapports sujet-espace afin d'identifier les mécanismes de localisation des untités dans les différents espaces et de spécifier le rôle des propriétés de l'espace dans le parcours des personnages.

Pour comprendre le rôle de l'espace dans le récit, la valeur symbolique des espaces créés et la signification de la localisation dans la création d'images qui invitent à penser l'espace d'une nouvelle façon il fallait envisager celui-ci dans sa complexité et reconstruire les itinéraires tracés dans le texte: espace initial, trajet, cible, et observer les phénomènes de concomitance et de superposition des espaces. Cette démarche a permis de spécifier, au niveau de la séquence narrative et au niveau global, les caractéristiques matérielles des lieux et les aspects de son occupation, les traits sémantiques des localisateurs et des expressions spatiales ainsi que leurs relations avec le contexte immédiat et global. Le dénominateur commun de ces nouvelles est un élément qui se reproduit de façon obsessionnelle: le passage ou le déplacement. Cortázar l'avait déjà dit: «c'est un élément qui nous met devant une fissure de la réalité à travers laquelle nous percevons une autre réalité, un autre ordre des choses, une série de lois qui ne sont pas moins rigoureuses que celles que nous appelons monde réel» ${ }^{6}$.

L'existence de deux espaces est nécessaire à la construction de la figure du passage, les personnages sont amenés à franchir un espace, certains réussissent totalement, (la lointaine), d'autres partiellement: «L'autre ciel», «L'île à midi». D'autres n'y arrivent pas.

6. Julio Cortázar, Entretiens avec Omar Prego (traducción de Françoise Rosset, Parr.s: Gallimard, 1986) 73. 


\section{Les concepts en opposition}

Les concepts spatiaux apparaissent dans le texte mis en relation par des rapports d'opposition: clos/ouvert, intérieur/ extérieur, prison/ liberté, malheur/bonheur, haut/bas.

Les espaces fermés, surtout la maison, loin d' être sécurisants, symbolisent la captivité, l'ennui. Les espaces ouverts: les rues, les passages, les ponts favorisent les déplacements et symbolisent l'accès à la liberté et au bonheur. L'espace quotidien est souvent opaque, il sert plutôt d'arrière-plan par rapport à l'espace évoqué qui est toujours le plus saillant. Les espaces intérieurs, quotidiens ne procurent ni la liberté, ni le bonheur, l'extérieur représente soit le bonheur soit le savoir, réponse à une question obsédante. Les frontières qui séparent ces espaces s'effacent pour permettre la réalisation du sujet.

En ce qui concerne l'activité du personnage, souvent le déplacement, le franchissement des frontières ne dépend pas de sa volonté, en cela, réside l'élément essentiel du fantastique cortazarien. Une force inconnue, fantastique fait agir les protagonistes, victimes d'une volonté qui leur échappe et des idées qui viennent de l'extérieur. Ils sont de ce côté et le défi consiste à passer de l'autre côté, les mouvements et l'orientation sont déterminés par une force extérieure qui les entraîne vers un univers proche et lointain à la fois.

Les personnages dans Pasajes sont souvent confrontés à l'existence d'une autre réalité. Ils sont envahis par des images d'un univers lointain qui s'installent dans leur quotidien, cet ailleurs les attire jusqu'à l'obsession, les conduit vers des espaces incertains. Incapables de maîtriser leur volonté, ils sont amenés à explorer ces zones. A la fin, aucun des personnages ne. parvient à rester dans l'espace évoqué de la façon dont il le rêvait. L'instabilité est un dénominateur commun de ces entités dont le parcour spatial est le même: refus de l'entourage immédiat, perception «d'autres côtés», recherche pour satisfaire un besoin de connaissance ou pour trouver l'environnement idéal, et passage. Mais leur localisation dans les 
espaces désirés ne dépend pas de leurs actes; les passages sont des événements subis et pas des actions contrôlées.

\section{Le passage ou la realisation du désir}

Le passage est un motif récurrent qui prend des formes variées et se manifeste comme un parcours sémantique qui constitue une métaphore fondatrice des récits. La manifestation textuelle de ce parcours implique un travail d'élaboration langagière assez complexe à cause du caractère fantastique qui lui est attribué.

Les personnages sont amenés à franchir un espace. Confrontés à l'existence d'une autre réalité qui les pousse à se déplacer, ils font le passage qui met en contact deux mondes. Ces passages se manifestent du point de vue linguistique de différentes façons. Parfois, l'absence d'intentionalité le caractérise comme événement, il implique un changement d'un état initial à un état distinct («El otro cielo»). Les agents sont des inanimés: les rues, les ponts, les galeries. Ce statut particulier de la causalité produit une illusion de causalité fantastique qui transgresse les lois ordinaires. Souvent le passage est à inférer, les traces linguistiques de la durée, des mouvements d'orientation, de direction, sont absentes. Par contre le caractère habituel est toujours marqué.

\section{La tâche du lecteur}

Tout au long de notre recherche, nous nous sommes interrogés sur le travail interprétatif du lecteur face aux nouvelles choisies. L'analyse du travail sur le langage opéré dans ces nouvelles met en évidence un nombre-important de difficultés interprétatives éventuelles (les aspects les plus troublants) et certaines caractéristiques de base du processus d'interprétation du sens.

L'analyse de cette problématique est restreinte aux opérations de base dans la compréhension des textes et à la spécificité du corpus 
choisi. Il ne s'agit pas d'un inventaire exhaustif des opérations cognitives, ni de la totalité des éléments qui rendent difficile la tâche interprétative, mais des problèmes essentiels dans les séquences qui présentent des points cruciaux concernant la construction de la représentation de la signification où les jeux référentiels sont poussés à l'extrêmc. A titre d'illustration nous presentons un exemple de structuration du récit où la connaissance du fonctionnement cognitif est mise à contribution pour programmer un parcours interprétatif determiné.

\section{Activation du schéma narratif}

Le texte joue sur la compétence du lecteur, notamment sur le présupposé de l'existence en mémoire d'un schéma de la suite événementielle d'un récit, et une capacité d'anticipation qui, en raison du type de texte lui permettra de faire des hypothèses sur la suite événementielle: cela le fait s'attendre à une cohérence logique. Or, dans les nouvelles de Cortázar, cette cohérence au niveau du racontant est souvent simulée.

La manipulation stratégique de la suite événementielle, l'exploitation du principe d'iconicité du récit, est un procédé évident dans «El otro cielo» et «La isla al mediodía». Cette disposition suit la logique du mouvement référentiel. La progression référentielle du domaine spatial, changement des espaces, voyage du protagoniste, autorise dans «L'île à midi», la construction de l'hypothèse du déplacement: «desembarcó» est pertinent pour répondre à la question «qu'est-ce qui se passe pour le protagoniste»?; le domaine référentiel en question est l'espace, le mouvement correspond à la progression logique des événements de la trame narrative. L'histoire est construite autour du désir du protagoniste de se rendre dans une petite île grecque. Le voyage est logiquement attendu comme une action clé de l'histoire et le lecteur accepte les pistes qui le conduisent à l'interprétation de cette action. 
D'autres difficultés trouvées concernent essentiellement l'activation de la mémoire de travail, la récupération de l'information, l'information partagée et l'univers de connaissances.

A cela, il faut ajouter un travail intense pour construire du sens à partir de: la transgression des principes de la construction des chaînes coréférentielles; des inférences; du traitement des indicateurs de la structuration textuelle; du problème de l'intertextualité, de l'interprétation des métaphores, et de la transgression du langage.

\section{Conclusions}

L'analyse des trois domaines référentiels dans ces quatre nouvelles nous a permis de montrer:

- la complexité du fonctionnement du dispositif textuel et la pertinence des choix théoriquesopérés aux niveaux linguistique et cognitif;

- le rôle des moyens linguistiques concernant la référenciation intervenant dans les jeux conceptuels avec le temps, l'espace et la personne pour produire l'imbrication des éléments doubles qui caractérisent le fantastique dans ces récits;

- les caractéristiques du traitement du langage opéré dans ces textes;

- les difficultés — au niveau cognitif - du traitement de l'information et la façon dont le texte programme les parcours interprétatifs possibles.

Interpréter le récit cortazarien implique la mise en oeuvre d'une démarche cognitive qui oblige à entreprendre une activité intense de traitement de l'information et à modifier pendant le temps de lecture ses stratégies habituelles de construction de la représentation, son univers de croyances, ses connaissances sur le monde. 